\title{
Antimonene-gold Based Twin-Core SPR Sensor with Side Polished Semi-Arc Groove Dual Sensing Channel: An Investigation with 2D Material
}

\section{Shivam Singh}

Motilal Nehru National Institute of Technology

Yogendra Kumar Prajapati ( $\nabla$ yogendrapra@gmail.com )

Motilal Nehru National Institute of Technology https://orcid.org/0000-0002-6752-5667

\section{Short Report}

Keywords: SSP-PCF, gold, antimonene, wavelength sensitivity, amplitude sensitivity.

Posted Date: November 15th, 2021

DOI: https://doi.org/10.21203/rs.3.rs-1057599/v1

License: (c) (1) This work is licensed under a Creative Commons Attribution 4.0 International License. Read Full License

Version of Record: A version of this preprint was published at Optical and Quantum Electronics on January 9th, 2022. See the published version at https://doi.org/10.1007/s11082-021-03505-7. 


\section{Antimonene-gold Based Twin-Core SPR Sensor with Side Polished Semi-Arc Groove Dual Sensing Channel: An Investigation with 2D Material}

Shivam Singh ${ }^{1}$, Yogendra Kumar Prajapati ${ }^{1}$

Abstract: We propose surface plasmon resonance (SPR) based single-side polished photonic crystal fiber (SSP-PCF) sensor for low as well as high refractive index (RI) sensing. To achieve this, an active metal gold (Au) is deposited on the PCF's flat narrow channels to form a dual-sensing channel. Following that, a thin nanolayer antimonene is deposited on $\mathrm{Au}$, as its buckled honeycomb lattice structure aids in the trapping of numerous biomolecules. For the sensing range of 1.27 to 1.39 , numerical results show that the wavelength sensitivity (WS) and amplitude sensitivity (AS) mounted on $77000 \mathrm{nmRIU}^{-1}$ and $1320.41 \mathrm{RIU}^{-1}$, respectively, with wavelength resolution (RW), and amplitude resolution (RA), as high as $1.298 \times 10^{-6} \mathrm{RIU}$, and $8.6 \times 10^{-7} \mathrm{RIU}$. The promising results obtained from the proposed SSP-PCF sensor offers improved refractive index sensing with a fine figure of merit (FOM), i.e., $311.74 \mathrm{RIU}^{-1}$ for the sensing range of 1.27 to 1.39 , which covers most known analytes such as proteins, cancer cells, glucose, viruses, DNA/RNA, medicinal drugs, halogenated organic acids. Further, the proposed sensor's design requires a simple fabrication procedure.

Keywords: SSP-PCF, gold, antimonene, wavelength sensitivity, amplitude sensitivity.

\section{Introduction}

Photonic crystal fiber (PCF) sensors based on surface plasmon resonance (SPR) technique has been widely used in the optical sensing applications. The sensor based on PCF-SPR is more favourable for use in the field of biosensors due to high sensitivity, fast response, and label free detection (R. Otupiri et al.2015). In the visible and near-infrared wavelengths, biosensors based on PCF-SPR are being investigated extensively (Y. Zhao et al. 2014; D. J. J. Hu et al. 2017; S. Chu et al. 2019). However, it is established and reported that, in the realm of sensing, the D-shaped (side polished) PCF-SPR fiber sensor is best suited because this sensor adopts external sensing mechanism, i.e., no need of filling or emptying the unknown sample of different refractive indices (RIs) in the selected air holes as used in the internal sensing based PCF sensors (M.A. Jabin et al.2019).

Y.K. Prajapati

yogendrapra@gmail.com

Shivam Singh

shivams20@gmail.com

${ }^{1}$ Department of Electronics and Communication Engineering, Motilal Nehru National Institute of Technology Allahabad, Prayagraj, UP 211004, India 
Further, the core mode and surface plasmon polariton (SPP) mode have a strong interaction because the SPP wave is close to the fiber core, therefore the sensitivity of the D-shaped PCF sensor is high over the conventional PCF-SPR based sensors(T. Huang et al. 2017; S. Singh et al.2020) . Recently, Chenguang Li et al. proposed a gold-coated Dshaped photonic quasi-crystal fiber RI sensor ( $\mathrm{Li}$ et al. 2019). By changing the gold layer thickness and air holes diameter, they have obtained the average and maximum wavelength sensitivities of $10250 \mathrm{nmRIU}^{-1}$ and $34000 \mathrm{nmRIU}^{-}$ ${ }^{1}$, respectively for the analyte RI range of 1.415 to 1.427 . In recent studies, it has been often seen that PCF structures have been designed mostly for high RI detection (i.e., equal or greater than 1.33). Further, the above discussed PCFSPR sensors have the limitation to detect low RI variation. This limitation has been overcome by using microchannel/two parallel channel based PCF-SPR sensors (E. Haque et al. 2019; F. Wang et al. 2018) and also offered the ease of fabrication process to realize them.

Till now, various external sensing-based configurations such as single and dual side polished (J.N. Dash et al. 2016; Shun Wang et al. 2019), D-shaped (S. Singh et al. 2021), and microchannel-based PCFs have been reported to reduce the structural complexity as well as to maximize the sensing performance. Akter et al. proposed an open-channel based low cost RI sensor (Akter et al.2019) in which they have used dual side gold coated open channels in such a way that the sensor could work in visible as well as NIR wavelength also. By varying the open channel radius and gold thickness, they have achieved high sensitivity and better resolution as $5000 \mathrm{~nm} / \mathrm{RIU}$ and $2.0 \times 10^{-5} \mathrm{RIU}$, respectively. Moreover, the reported sensor also showed figure of merit (FOM) as $47 \mathrm{RIU}^{-1}$ within the RI ranged from 1.33 to 1.39 .

As of late, various new optical materials are now explored and also exploited to enhance the sensing performance of a PCF based sensor (R. Chauhan et al. 2016). In this regard, different 2D optical materials such as graphene, molybdenum disulfide $\left(\mathrm{MoS}_{2}\right)$, tungsten diselenide $\left(\mathrm{WSe}_{2}\right)$, and tungsten disulfide $\left(\mathrm{WS}_{2}\right)$ have been widely used $(\mathrm{K}$. S. Novoselov et al.2004; C. Zhu et al. 2015; X.Gu et al.2016; F. Wang et al.2017). Among these, graphene and $\mathrm{MoS}_{2}$ are frequently exercised. Graphene shows good optical conductivity in the near and mid-IR spectrum. But it is a semimetal with zero bandgap which restricts its emphatic use in some optical devices. On the other hand, $\mathrm{MoS}_{2}$ has a tunable band gap but, its band gap tunability is below $1.9 \mathrm{eV}$ which may also restrain its effective utilization in photonic devices. Therefore, to mitigate these major issues, recently, researchers have reported antimonene, a newly discovered 2D material. It consists of $\mathrm{SP}^{3}$ - hybridized thin nanolayer sheet of antimony atoms (S. Lee et al. 2014; G. Wang et al. 2015). Moreover, its large bandgap swing (i.e., from 0 to 2.62eV) gives it an extra edge over graphene and $\mathrm{MoS}_{2}$. Yan Shao et al. reported the experimental work based on the epitaxial growth of monolayer antimonene (Yan Shao et al. 2018). Its atomically thin layer was grown on silver substrate. After experimental realization, they have found that few-layer or monolayer antimonene can be a suitable optical material for the applications in optoelectronic and nanodevices. Tianyu Xue et al. reported an antimonene-based SPR sensor for the detection of miRNA with detection limit of 10aM (T. Xue et al.2019).

In this paper, Finite Element Method (FEM) is applied to an external sensing-based symmetrical core SSP-PCF SPR sensor along with novel 2D material antimonene for the detection of low and high analyte RIs, inspired by previous research and the need to improve the performance of PCF-SPR based sensor. The novelty of this work is two-fold. First, we have proposed a single side polished (Quasi D-shaped) fiber. In such fiber, the core region becomes 
much nearer to the polished surface due to which a strong coupling connectivity between core mode energy and surface energy (SPP) is established. Second, dual sensing-channel emerged due to a single micro-opening reduces excess coating of plasmonic material, and made the proposed sensor economical for sensing experimentation. The results reveal that when coupling of the surface plasmon polariton (SPP) and the core mode is proven, the sensitivity improves.

\section{Structural design and realization possibility}

\subsection{Structural design and working principal}

The stacked preform and 2D schematic view of the stated SSP-PCF sensor is depicted in Fig.1 (a-b) while Fig.1(c) displays the 3D schematic model in which two narrow sensing channels are separated from each other by a single arcshaped micro-opening. The sensing layer holds bio-liquid (containing biomolecules) based analytes whose refractive index varies from 1.27 to 1.39. To detect these analytes, gold, and antimonene have been coated on flat fiber surfaces. Here, antimonene is used as bio-recognition element (BRE), because it strongly involves in the bulk adsorption of the biomolecules. It happens due to the better interaction of antimonene's hexagonal lattice frame with the biomolecules having a carbon ring-based structure.

The proposed structure of the SSP-PCF sensor is made up with two square rings (inner and outer) consisting of circular air holes. Four air holes from the top of the second square ring have been completely omitted in order to create a flat surface, while a single circular air hole placed at the top middle position of this ring has been partially removed to make a semi-arc groove (micro-opening) with an arc radius (R) of 1.6 $\mu \mathrm{m}$. A single top flat surface of the fiber has been divided into two independent flat surfaces due to micro-opening Further, the gold and antimonene layers have been coated over these two flat surfaces to make them as a dual sensing channel. We have also indicated the sensing medium in Fig.1 (b) (in sky blue color) above these layers. This sensing medium includes bio-molecules in the form of sample/analyte of different refractive indices.
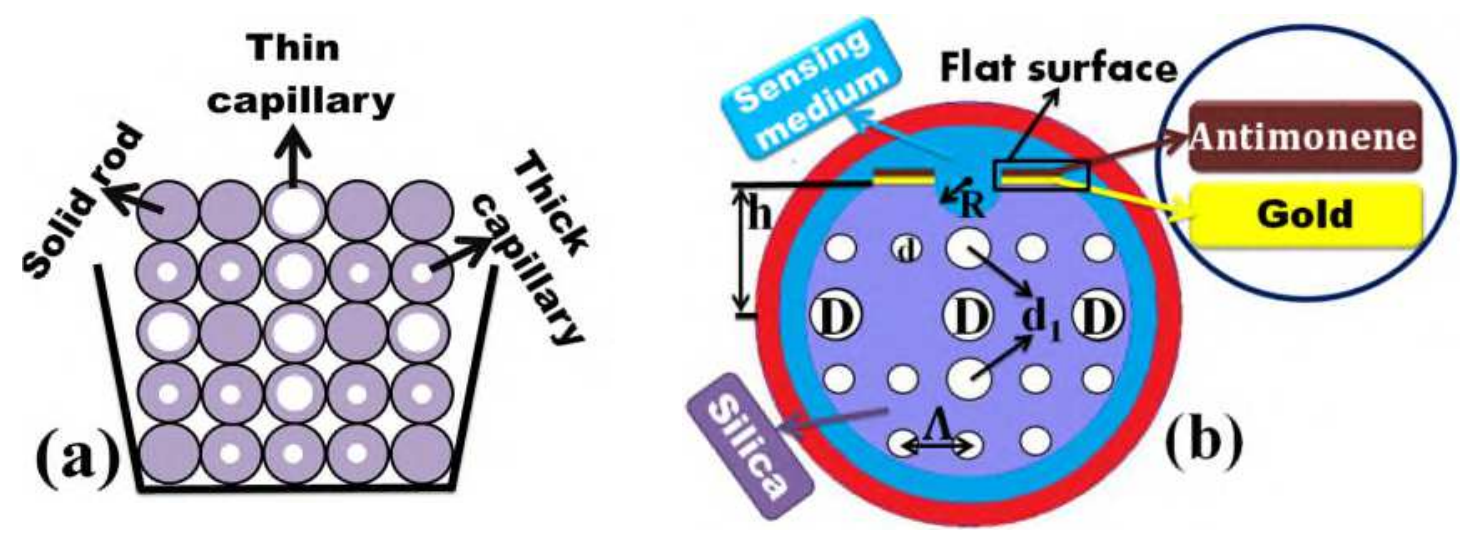

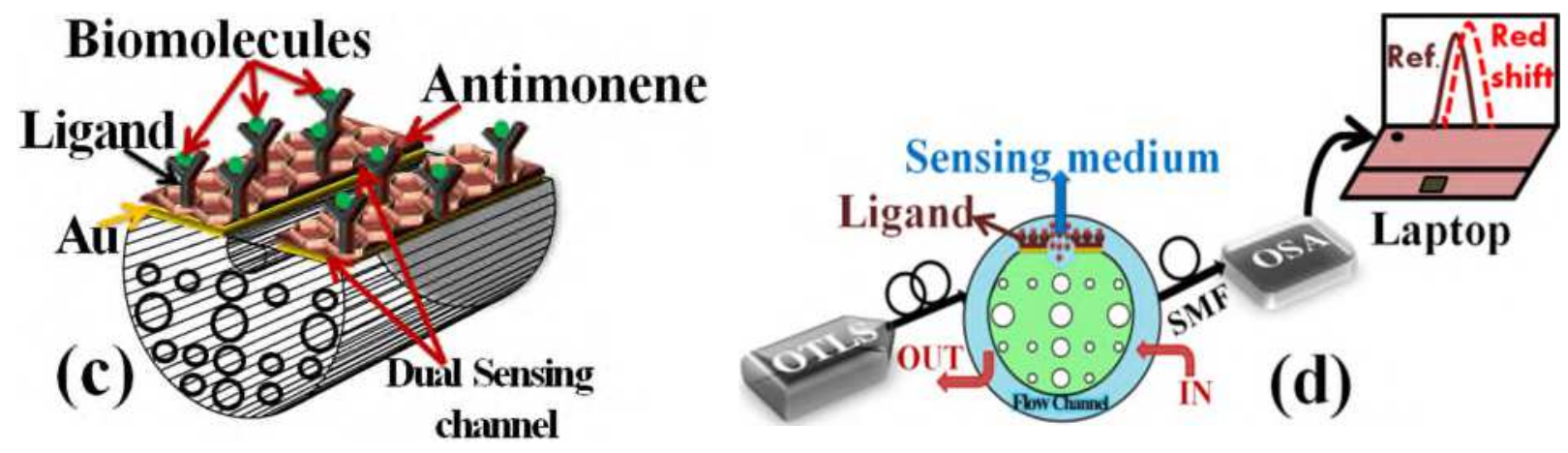

Fig.1. (a) Stacked preform and, (b) 2D cross-section of the proposed SSP- PCF with enlarged view of the metal layers (c) 3D schematic model of the proposed PCF sensor with biomolecules affinity layers of gold ( $\mathrm{Au}$ ) and antimonene (d) Schematic arrangement for the experimental realization of the proposed sensor.

For simulation, RIs and thickness of used materials like silica, gold and antimonene are listed in Table 1 for the proposed SSP-PCF sensor, which can be obtained as follows (E.K. Akowuah et al. 2012; RK Gangwar et al. 2019; Deobrat Singh et al.2016).

$\varepsilon_{A u}=1-\frac{\lambda^{2} \lambda_{c}}{\lambda_{p}^{2}\left(\lambda_{c}+j \lambda\right)}$

Where, $\lambda_{c}$ and $\lambda_{p}$ are the bulk collision and plasma wavelengths whose values are given as $8.9342 \mu \mathrm{m}$ and 0.16826 $\mu \mathrm{m}$, respectively. The wavelength sensitive refractive index of antimonene can be estimated using (Deobrat Singh et al.2016).

Table 1: Configuration of the proposed SSP-PCF sensor

\begin{tabular}{|c|c|c|c|}
\hline $\begin{array}{c}\text { Structure } \\
\text { composition }\end{array}$ & Used materials & $\begin{array}{c}\text { Wavelength dependent RI } \\
\left(\mathbf{n}+\mathbf{i}^{*} \mathbf{k}\right)\end{array}$ & Thickness \\
\hline $1^{\text {st }}$ layer & PCF made from silica & E.K. Akowuah et al. 2012 & - \\
\hline $2^{\text {nd }}$ layer & Gold $(\mathrm{Au})$ & RK Gangwar et al. 2019 & $\mathrm{Au}=40 \mathrm{~nm}$ \\
\hline $3^{\text {rd }}$ layer & $\begin{array}{c}\text { Antimonene } \\
\text { Monolayer }(\mathrm{L})\end{array}$ & Deobrat Singh et al.2016 & $\mathrm{L}=0.16 \mathrm{~nm}$ \\
\hline $4^{\text {th }}$ layer & Analyte $\left(\mathrm{n}_{\mathrm{a}}\right)$ & $\mathrm{Re}\left(\mathrm{n}_{\mathrm{a}}\right)=1.27$ to 1.39 & - \\
\hline
\end{tabular}

Further, to strengthen the coupling between fundamental and SPP modes, four air holes of the first square ring have been scaled down to a diameter (d) value of $1 \mu \mathrm{m}$. Dual-core has been created by completely eliminating the two air holes placed horizontally to the central air hole of diameter (D) $2.5 \mu \mathrm{m}$ of the first square ring. The other two air holes of diameter $\left(\mathrm{d}_{1}\right) 1.8 \mu \mathrm{m}$ in the same ring are placed vertically to the central air hole. To absorb dispersed radiation, an artificial layer called perfectly matched layer (PML) with a thickness of $\mathrm{T}=10.4 \mu \mathrm{m}$ has been fixed on the exterior 
wall of the PCF. Fig.1 (d) displays an organized experimental set up to initiate a sensing environment for the proposed sensor. The electromagnetic (EM) wave transmits through the single-mode fiber (SMF) with the help of an optically tunable laser source (OTLS). A narrow circular passage around the outer wall of the fiber is used to maintain the flow of liquid/analyte. When the EM wave reaches to a dielectric-metal interface, the ligands of gold/ antimonene coated surface interacts with the analyte and causing a shift in resonant position. The obtained shift can be visualized on the screen with the help of an optical spectrum analyzer (OSA).

\subsection{Sensing parameters of a proposed SSP-PCF Sensor}

\subsubsection{Mode confinement loss:}

The mode confinement/propagation loss $\left(\alpha_{\text {loss }}\right)$ in $x$-and $y$-polarization direction can be defined as follows (J.N. Das et al. 2018):

$\alpha_{\text {loss }}(\mathrm{dB} / \mathrm{cm})=8.686 \times 2 \pi / \lambda \times \operatorname{Imag}\left(\mathrm{n}_{\text {eff }}\right) \times 10^{4}$

Where, $\operatorname{Imag}\left(\mathrm{n}_{\text {eff }}\right)$ is the imaginary part of the effective RI. The unit of $\left(\alpha_{\text {loss }}\right)$ is $\mathrm{dB} / \mathrm{cm}$.

\subsubsection{Wavelength sensitivity:}

The wavelength sensitivity (WS) can be expressed (M.R.Hasan et al. 2018;S. Singh et al. 2019) as:

$\mathrm{S}_{\mathrm{W}}=\partial \lambda_{\mathrm{r}} / \partial \mathrm{n}_{\mathrm{a}}$

Where $\lambda_{\mathrm{r}}$ is the resonance wavelength, $\mathrm{n}_{\mathrm{a}}$ is the RI of the analyte/biomolecules and $\partial \lambda_{r}, \partial n_{a}$ denotes shift in resonance wavelength and analyte $\mathrm{RI}$. The unit of the wavelength sensitivity is $\mathrm{nmRIU}^{-1}$.

\subsubsection{Amplitude sensitivity:}

The response of amplitude sensitivity (AS) can be calculated (E. Haque et al. 2018; M.R.Hasan et al. 2018) as:

$\mathrm{S}_{\mathrm{Amp}}(\lambda)=-1 / \alpha\left(\lambda, \mathrm{n}_{\mathrm{a}}\right) \partial \alpha\left(\lambda, \mathrm{n}_{\mathrm{a}}\right) / \partial \mathrm{n}_{\mathrm{a}}$

The unit of the amplitude sensitivity is $\mathrm{RIU}^{-1}$.

\subsubsection{Figure of Merit:}

The figure of merit (FOM) is an essential parameter for proposed sensor and can be defined (S. Singh et al. 2020) as:

$\mathrm{FOM}=\frac{\mathrm{S}_{\mathrm{W}}}{\mathrm{FWHM}}$

The unit of FOM is RIU-1.

\subsection{Realization possibility}


The fabrication of proposed SSP-PCF sensor can begin with the stack and draw approach (R. Zakaria et al. 2017), which involves bundling the different capillaries and solid rods. The circular air holes can be scaled to different sizes with thin and thick capillaries. With thin and thick capillaries, the circular air holes can be adjusted to different sizes. A thin capillary will be picked and polished to a specific extent to create a micro-opening on the top smooth surface. After completion of the PCF fabrication process, few layers antimonene nanosheets can be drawn out using the liquidphase sonification method by unrooting the feeble van der Waals forces between the bulk antimony atoms (M. Assebban et al. 2020). Then, nanosheets of antimonene can be assembled on Au coated fiber surface using the existing layer-by-layer film deposition technique (R. Zakaria et al. 2017; M. Assebban et al. 2020). Therefore, it is believed that the proposed SSP-PCF sensor can be practically realized using the existing techniques.

\section{Simulation Results and Discussion}

This section devoted to simulation result analysis of the propounded SSP-PCF model. All simulated results reveal that the sensing performance has been analyzed by mode field distributions as well as geometrical and, material parameter variations. Fig. 2 (a), blue and green lines indicate the change in effective RIs of the fundamental and surface plasmon polariton (SPP) mode while red curve shows loss incurred due to light propagation via core. It can be clearly observed that both blue and green lines moving downward with wavelength and intersects with each other where the real parts of effective RIs of both modes (i.e., core and SPP) become equal. Due to this, resonance occurs and the generated evanescent field penetrates well into both metal layers and analyte, results a sharp resonance peak $\left(\lambda_{\mathrm{r}}\right)$ obtained at $1.77 \mu \mathrm{m}$. Also, it is observed from the figure that there are two polarization modes, i.e., even $x$-polarized mode and the odd $x$-polarized mode. The coupling loss in even $x$-polarized direction is much higher than that in odd $x$-polarized direction.

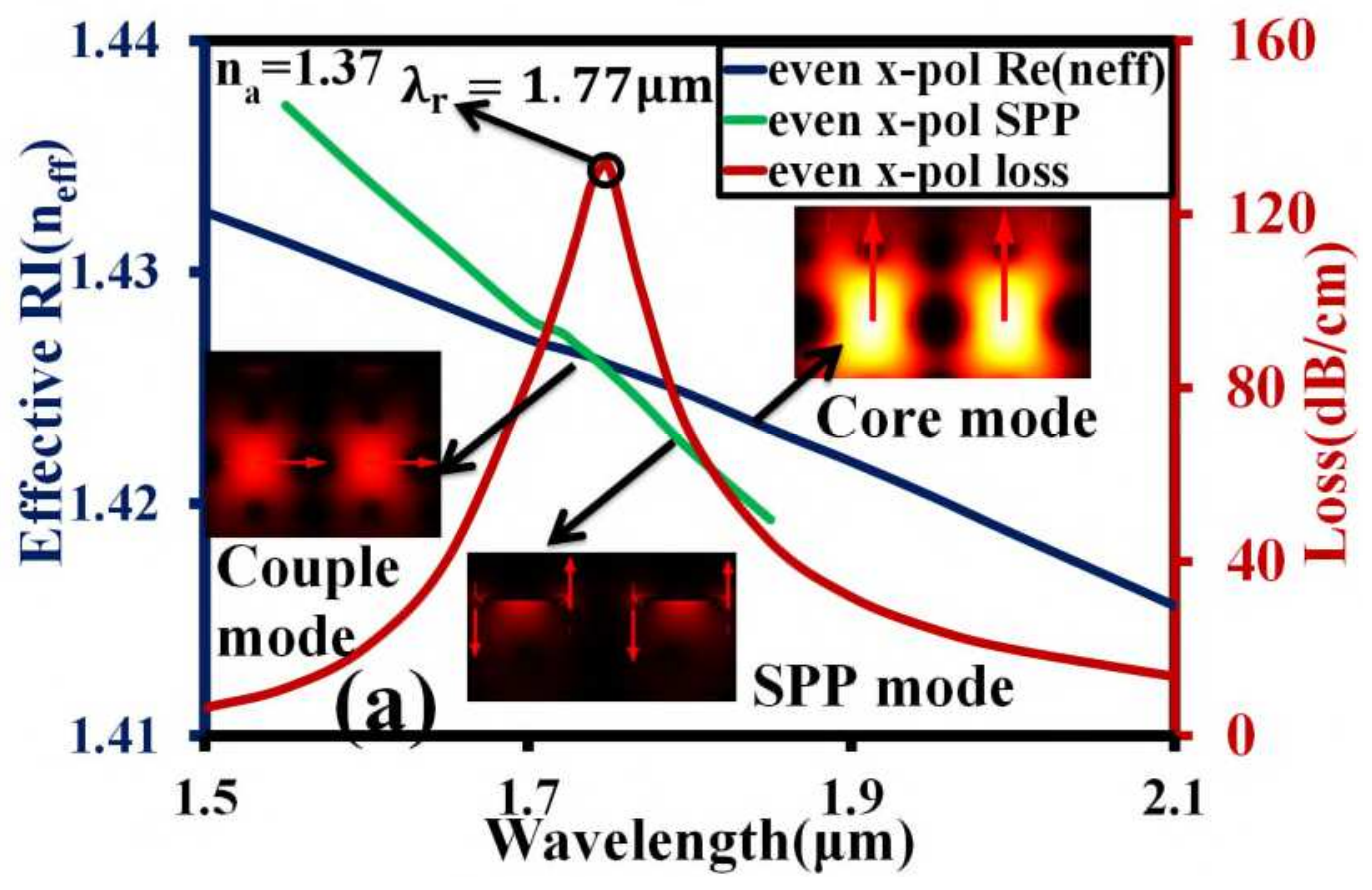



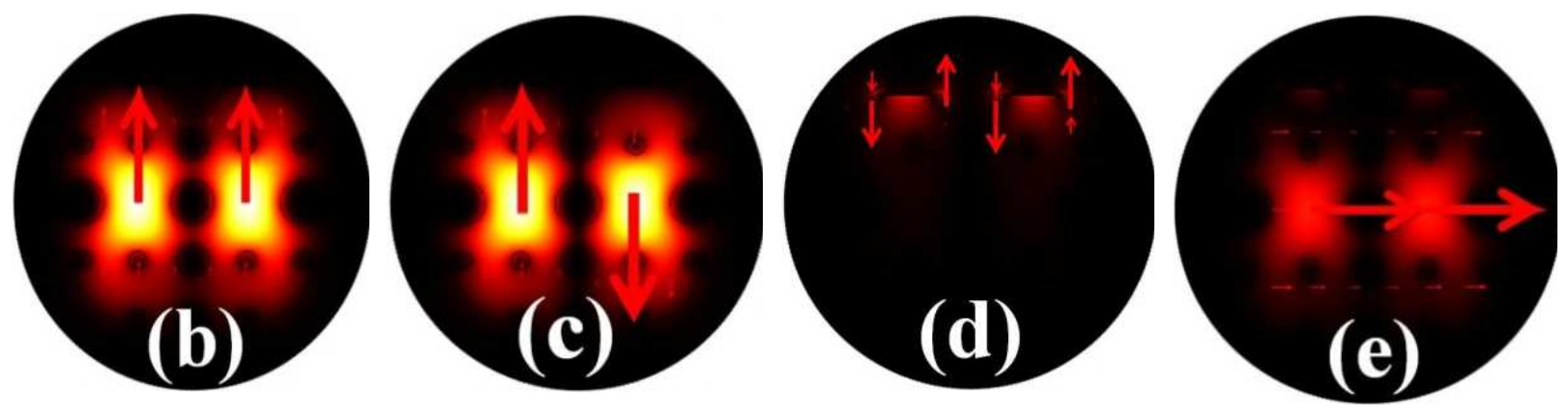

Fig.2. (a) Loss curve and dispersion relations between core and SPP mode. Field distributions at (b) even and, (c) odd $x$-polarized core mode (d) SPP mode (e) coupling mode When $\mathrm{Au}=40 \mathrm{~nm}, \mathrm{~L}=3, \mathrm{D}=2.5 \mu \mathrm{m}, \mathrm{d}=1 \mu \mathrm{m}, \mathrm{d}_{1=1} 1.8 \mu \mathrm{m}$, $\mathrm{R}=1.6 \mu \mathrm{m}, \mathrm{h}=6.4 \mu \mathrm{m}, \Lambda=3.2 \mu \mathrm{m}$ and $\mathrm{T}=10.4 \mu \mathrm{m}$.

Fig. 2(b-c) shows the electric field dispensation for even and odd $x$-polarization in which the entire field is confined within the core with high intensity. Fig. 2(d) displays that the core directed electric field relocates its energy to the dielectric-metal interface in the form of surface plasmon waves. The strength of these waves measures the sensitivity level on the metallic surface. Fig.2 (e) resembles the field profile when the phase-matching condition achieved. At this condition, maximum energy appears at the interface as the analyte RI impacts well to the real portion of effective RI of SPP mode.

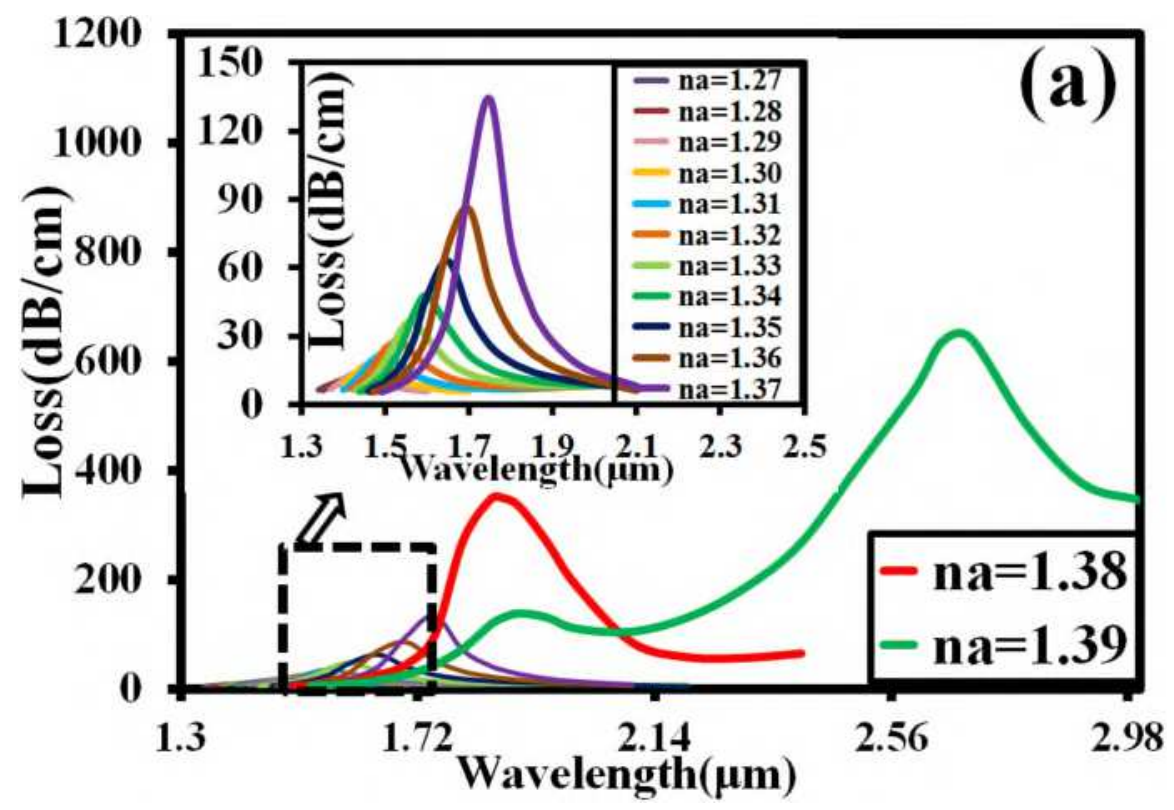

It is observed in Fig. 3(a) that the first coupling peak is obtained at $1380 \mathrm{~nm}$ for analyte RI 1.27. Then, the peak shifts to $2650 \mathrm{~nm}$ for analyte RI 1.39. This happens as the real part of SPP mode RI varies with the variation in analyte RI and causing shift in resonant wavelength position. The highest shift of $770 \mathrm{~nm}$ is obtained for the change in analyte RI from 1.38 to 1.39 . Therefore, the maximum WS and RI resolution are obtained as $77000 \mathrm{~nm} / \mathrm{RIU}$ and $1.298 \times$ 
$10^{-6}$ RIU. Apart from wavelength sensitivity, change in analyte RI can also be sensed with amplitude sensitivity (AS) response, shown in Fig.3 (b). The maximum AS is found as 1320.41 RIU $^{-1}$ at $1.95 \mu \mathrm{m}$ for $\mathrm{n}_{\mathrm{a}}=1.37$. Fig.3(c) displays the fitting polynomial as well as wavelength sensitivity variation with the change in analyte RI from 1.27 to 1.39 . An adjustable $\mathrm{R}^{2}$ value of 0.9966 is obtained which shows better fitting linearity. Additionally, the wavelength sensitivity variation also shows a linear response with analyte RI.
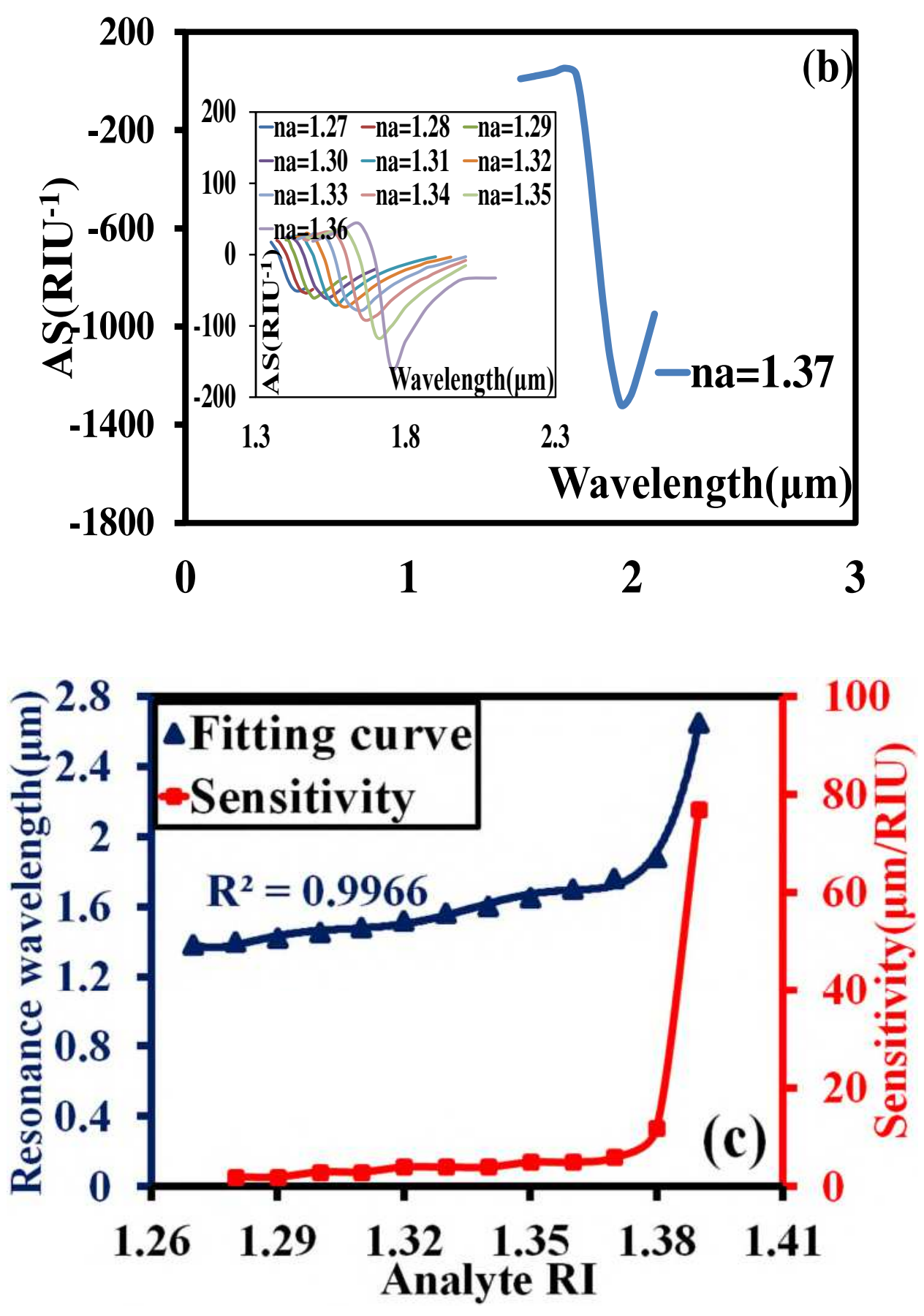
Fig.3. (a) Loss spectra and, (b) amplitude sensitivity response (c) fitting polynomial with WS variations for the change in analyte RI from 1.27 to 1.39 , respectively.

Fig. 4(a) displays the impact of gold thickness on the sensor's performance. The loss peak with its decreasing value shifts towards the lower wavelength with an increase in Au thickness. It happens because a thick Au layer produces higher damping loss and causing low field penetration in its surrounding medium. The maximum loss is obtained as $138.96 \mathrm{~dB} / \mathrm{cm}$ at $1.77 \mu \mathrm{m}$ for gold $(\mathrm{Au})$ thickness $=40 \mathrm{~nm}$ and $\mathrm{n}_{\mathrm{a}}=1.37$. A change in the number of antimonene layers ( $\mathrm{L}=1$ to $\mathrm{L}=4$ ) affects the resonant position, shown in Fig. 4(b). With monolayer $(\mathrm{L}=1)$, it shows a weak coupling between fundamental and SPP mode. Therefore, the obtained loss is minimum (i.e., $126.42 \mathrm{~dB} / \mathrm{cm}$ ). But, when the number of layers increases, the coupling intensity increases which results in a higher loss. It happens because when the antimonene layer becomes thick, it's buckled atomic lattice structure traps maximum light energy in the vicinity of the metal surface. Therefore, the maximum coupling loss achieved as $178.37 \mathrm{~dB} / \mathrm{cm}$ at $1.77 \mu \mathrm{m}$. A little variation in the resonant position can be observed in Fig.4(c-d) and (e) for the change in diameters of the large, medium and small air hole which shows that the proposed PCF sensor is quite robust against the variation in its geometrical parameters. Moreover, the resonant point appeared at the same wavelength against the variation in PML thickness, shown in Fig. $4(f)$.
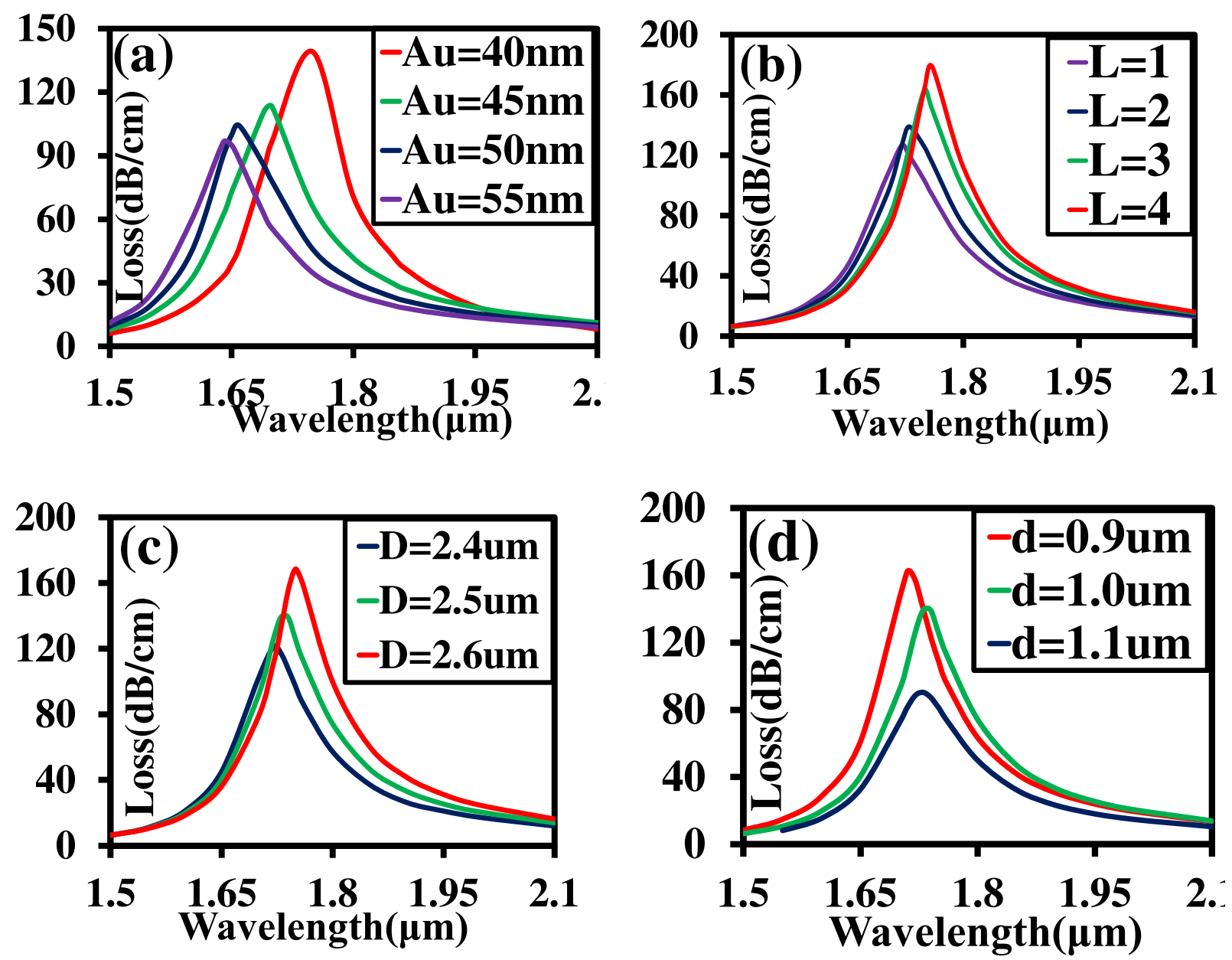

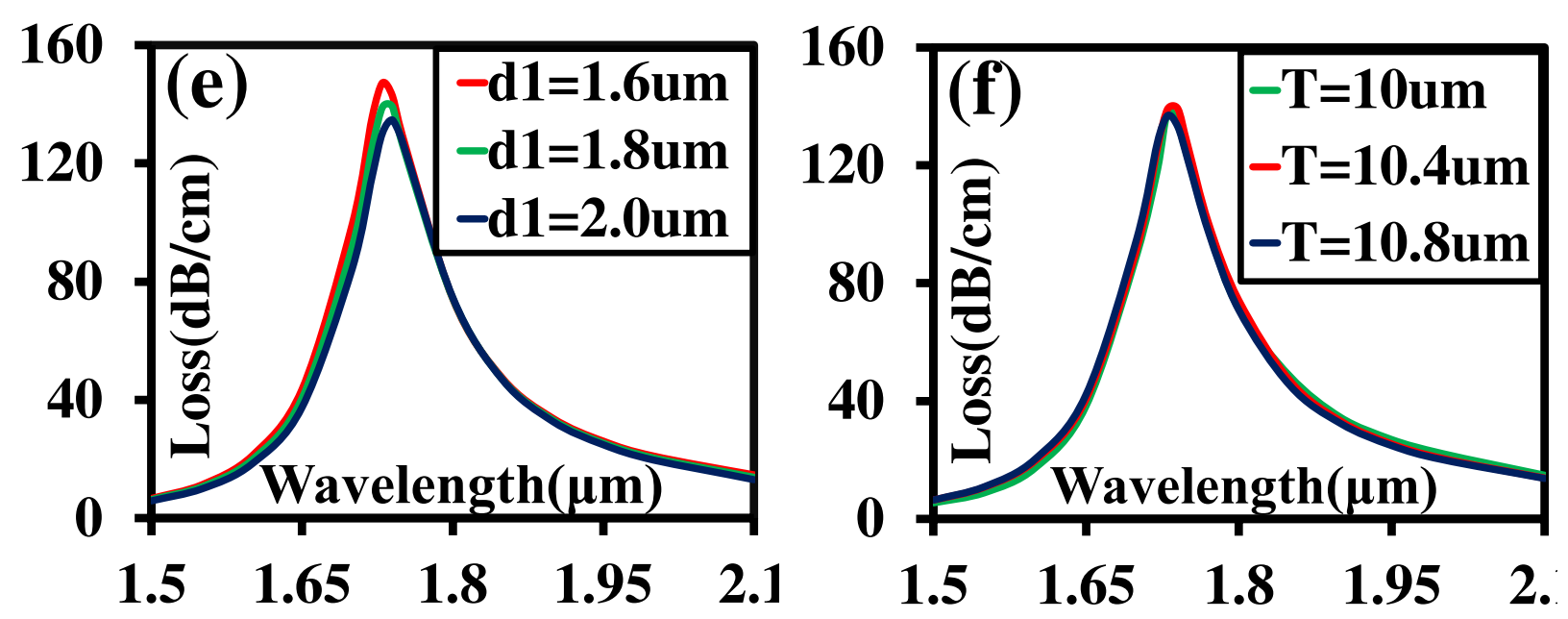

Fig.4. Variation in resonant wavelength with change in (a) Au thickness, (b) number of antimonene layer, (c) large, (d) medium, (e) small air hole diameter, and (f) PML thickness.

A change in arc radius ( $\mathrm{R}$ ) also affects the coupling loss, depicted in Fig.5 (a). At $\mathrm{R}=1.7 \mu \mathrm{m}$, the coupling loss reaches to the minimum value of $121.68 \mathrm{~dB} / \mathrm{cm}$ as gold coated sensing channel becomes narrower. But, when the radius reaches its minimum value ( $\mathrm{R}=1.5 \mu \mathrm{m})$, coupling loss increases and reached to its maximum value of $153.47 \mathrm{~dB} / \mathrm{cm}$. It happens because lowering the radius offers a broad sensing channel that supports an intensive field near the metal surface.

In Fig.5 (b), a significant shift in the resonance wavelength can be observed while changing the polishing height (h) measured from middle of the fiber core axis to the polished surface. The optimized value of polishing height from the central core has been taken as $\mathrm{h}=6.4 \mu \mathrm{m}$. It can be seen from Fig. 5(b) that increasing the height causes a decrease in the loss value while it drastically increases when the polishing height reduces. It happens because when the height gets detracted, the polished surface becomes closer to the core and supports the coupling between core and SPP mode. Fig.5(c) depicts the coupling loss dependency with the change in pitch size. When the lattice spacing between the consecutive air holes gets reduced, the loss becomes higher and obtained as $268.61 \mathrm{~dB} / \mathrm{cm}$ at $\Lambda=3.1 \mu \mathrm{m}$. Whereas, it decreases with increasing pitch size and obtained as $139.18 \mathrm{~dB} / \mathrm{cm}$ and $88.97 \mathrm{~dB} / \mathrm{cm}$ at pitch size $3.2 \mu \mathrm{m}$ and $3.3 \mu \mathrm{m}$, respectively. It happens because increasing the pitch size reduces the RI difference between core and cladding of the proposed structure. Apart from exploring all the possible performance parameters, full width at half maximum (FWHM) also plays an important role while designing PCF based sensor. A large value of FWHM causes uncertainty in picking out the exact resonant point which may result in false positive detection. Therefore, the PCF sensor having low FWHM is highly recommended. In addition, with the help of FWHM, the FOM is plotted in Fig. 5 (d). Fig.5 (d) displays the FOM variation in accordance with analyte RI. In Fig.5(d), the FOM value varies in between 14 to 315 $\mathrm{RIU}^{-1}$ and found maximum as $311.74 \mathrm{RIU}^{-1}$ at $\mathrm{n}_{\mathrm{a}}=1.38$. 

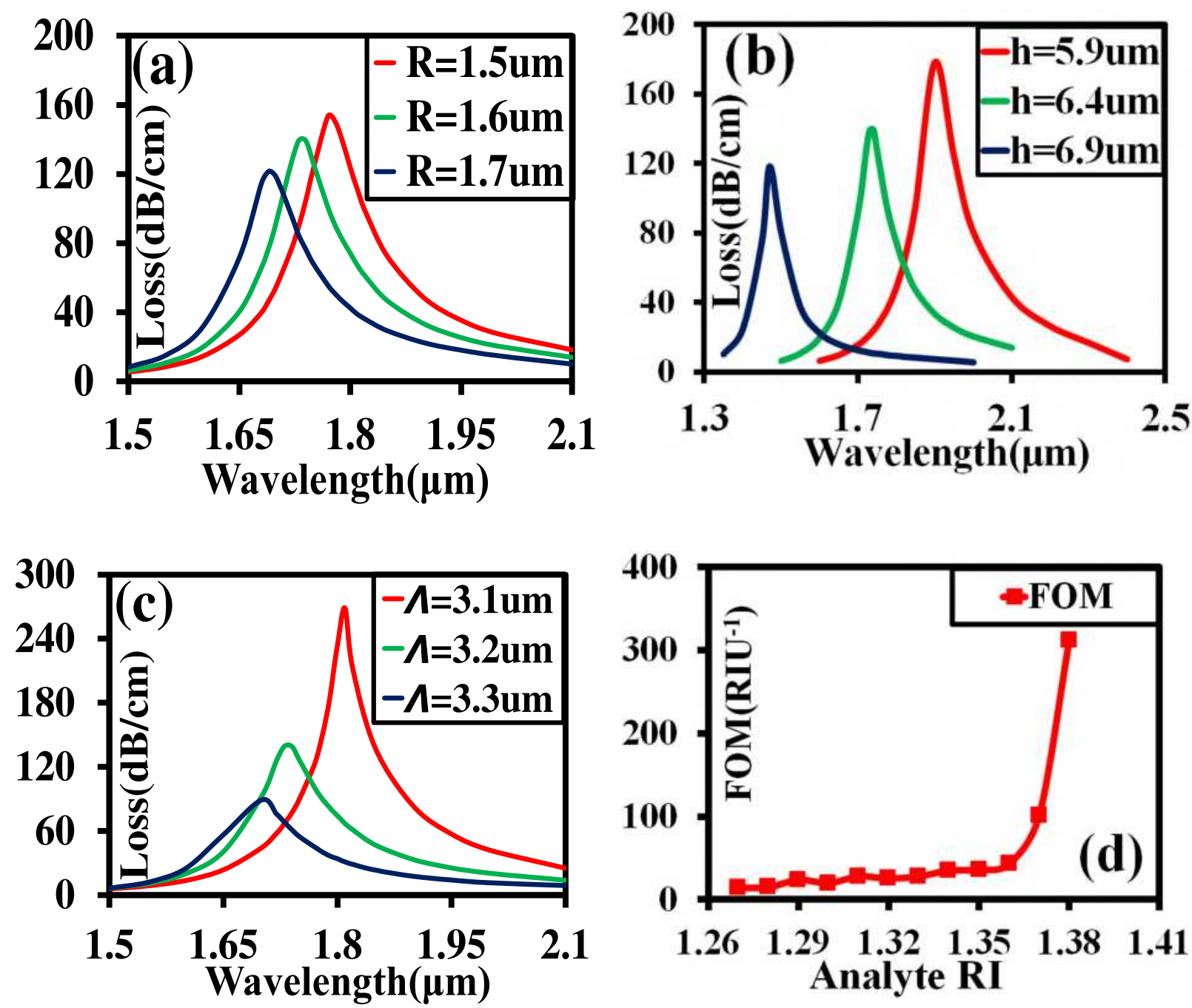

Fig.5. Change in loss peak with the variation in (a) Open arc radius, (b) polishing depth and, (c) pitch size (d) FOM variation with analyte RI.

Table 2 verifies the numerical evaluation of all the major performance parameters for the proposed SSP-PCF sensor. It can be seen from above table that the sensitivity increases at a slow pace with analyte RI for both even and odd $x$ polarized mode. Here, we have considered even $x$-polarized mode. Therefore, the obtained wavelength sensitivities are 2000, 2000, 3000, 3000, 4000, 4000, 4000, 5000, and 5000, 6000 and 12000nm/RIU, respectively for analyte RI ranges from 1.27 to 1.38 . Then, a sudden hike in the sensitivity as $77000 \mathrm{~nm} / \mathrm{RIU}$ can be observed when the analyte RI changes from 1.38 to 1.39 . The same phenomenon can be also observed for the amplitude sensitivity in which the sensitivity gradually increases as $51.16,53.8,60.2,60.58,71.1,73.27,78.83,86.1,113.57$ and 157.66 RIU $^{-1}$, respectively for the change in analyte RI from 1.27 to 1.36. Finally, it reaches to a maximum (max.) value of 1320.41 $\mathrm{RIU}^{-1}$ at $\mathrm{n}_{\mathrm{a}}=1.37$. Similarly, the wavelength resolution $\left(\mathrm{R}_{\mathrm{w}}\right)$ and amplitude resolution $\left(\mathrm{R}_{\mathrm{A}}\right)$ are also shown in the above Table 2. The values of both Rw and $\mathrm{R}_{\mathrm{A}}$ are found as finer as $1.298 \times 10^{-6} \mathrm{RIU}$ and $8.6 \times 10^{-7} \mathrm{RIU}$ at analyte RI 1.38 and 1.37 . 
Table 2: Performance evaluation of the proposed SSP-PCF sensor in terms of sensitivity and resolution for the change in analyte RI from 1.27 to 1.39

\begin{tabular}{|c|c|c|c|c|c|c|c|c|c|c|}
\hline \multirow[t]{2}{*}{$\mathbf{n}_{\mathrm{a}}$} & \multicolumn{2}{|c|}{$\begin{array}{c}\text { Resonant } \\
\text { peak } \\
\lambda_{\mathrm{r}}(\mathrm{nm})\end{array}$} & \multicolumn{2}{|c|}{$\begin{array}{c}\text { Shift in peak } \\
\Delta \lambda_{r}(\mathrm{~nm})\end{array}$} & \multicolumn{2}{|c|}{$\begin{array}{c}\text { Wavelength } \\
\text { sensitivity } \\
\text { WS (nm/RIU) }\end{array}$} & \multicolumn{2}{|c|}{$\begin{array}{l}\text { Amplitude } \\
\text { sensitivity } \\
\text { AS }\left(\text { RIU }^{-1}\right)\end{array}$} & \multirow{2}{*}{$\begin{array}{c}\begin{array}{c}\text { Wavelength } \\
\text { Resolution } \\
\text { Rw (RIU) }\end{array} \\
\text { Even } \\
x \text {-pol }\end{array}$} & \multirow{2}{*}{$\begin{array}{c}\begin{array}{c}\text { Amplitude } \\
\text { Resolution } \\
\text { R }_{\text {A }}(\text { RIU) }\end{array} \\
\text { Even } \\
x \text {-pol }\end{array}$} \\
\hline & $\begin{array}{l}\text { Even } \\
x \text {-pol }\end{array}$ & $\begin{array}{l}\text { Odd } \\
x \text {-pol }\end{array}$ & $\begin{array}{l}\text { Even } \\
x \text {-pol }\end{array}$ & $\begin{array}{l}\text { Odd } \\
x \text {-pol }\end{array}$ & $\begin{array}{l}\text { Even } \\
x \text {-pol }\end{array}$ & $\begin{array}{l}\text { Odd } \\
x \text {-pol }\end{array}$ & $\begin{array}{l}\text { Even } \\
x \text {-pol }\end{array}$ & $\begin{array}{l}\text { Odd } \\
x \text {-pol }\end{array}$ & & \\
\hline 1.27 & 1380 & 1380 & 20 & 20 & 2000 & 2000 & 51.1 & 50.5 & $5 \mathrm{E}-5$ & $3.3 \mathrm{E}-5$ \\
\hline 1.28 & 1400 & 1400 & 20 & 20 & 2000 & 2000 & $\begin{array}{l}53.8 \\
\end{array}$ & 52.3 & $5 \mathrm{E}-5$ & $1.3 \mathrm{E}-5$ \\
\hline 1.29 & 1420 & 1420 & 30 & 30 & 3000 & 3000 & 60.2 & 58 & $3.3 \mathrm{E}-5$ & $1.81 \mathrm{E}-4$ \\
\hline 1.30 & 1450 & 1450 & 30 & 30 & 3000 & 3000 & 60.5 & 62.5 & $3.3 \mathrm{E}-5$ & $9.5 \mathrm{E}-6$ \\
\hline 1.31 & 1480 & 1480 & 40 & 35 & 4000 & 3500 & 71.1 & 70.2 & $2.5 \mathrm{E}-5$ & $4.6 \mathrm{E}-5$ \\
\hline 1.32 & 1520 & 1515 & 40 & 45 & 4000 & 4500 & 73.2 & 74.3 & $2.5 \mathrm{E}-5$ & $1.8 \mathrm{E}-5$ \\
\hline 1.33 & 1560 & 1560 & 40 & 45 & 4000 & 4500 & 78.8 & 77.8 & $2.5 \mathrm{E}-5$ & $1.37 \mathrm{E}-5$ \\
\hline 1.34 & 1600 & 1605 & 50 & 45 & 5000 & 4500 & 86.1 & 85.5 & $2 \mathrm{E}-5$ & $3.7 \mathrm{E}-6$ \\
\hline 1.35 & 1650 & 1650 & 50 & 60 & 5000 & 6000 & 113.5 & 110 & $2 \mathrm{E}-5$ & $2.24 \mathrm{E}-6$ \\
\hline 1.36 & 1700 & 1710 & 60 & 60 & 6000 & 6000 & 157.6 & 160.1 & $1.6 \mathrm{E}-5$ & $8.6 \mathrm{E}-7$ \\
\hline 1.37 & 1760 & 1770 & 120 & 105 & 12000 & 10500 & 1320 & 1298 & $8.3 \mathrm{E}-5$ & - \\
\hline 1.38 & 1880 & 1875 & 770 & 765 & 77000 & 76500 & - & - & $1.29 \mathrm{E}-6$ & - \\
\hline 1.39 & 2650 & 2640 & - & - & - & - & - & - & - & - \\
\hline
\end{tabular}

At this stage, it is important to compare the performance of proposed SSP-PCF sensor with the D-type PCF sensors available in the state-of-the-art. For this comparison, a common performance parameter is chosen as the wavelength and amplitude sensitivities, wavelength and amplitude resolutions and arranged in Table 3. The table clearly reveals that all performance parameters are superior to state-of-the-art research except amplitude sensitivity reported in M. R. Hasan et al. 2018.

Table 3: A brief comparison of the proposed SSP-PCF with newly reported plasmonic RI sensors

\begin{tabular}{|c|c|c|c|c|c|c|c|}
\hline $\begin{array}{c}\text { Refs. / } \\
\text { year }\end{array}$ & $\begin{array}{c}\text { RI } \\
\text { range }\end{array}$ & $\begin{array}{c}\text { Geometric } \\
\text { structure }\end{array}$ & $\begin{array}{c}\text { Used } \\
\text { material }\end{array}$ & $\begin{array}{c}\text { Max. } \\
\text { Wavelength } \\
\text { sensitivity } \\
\text { (nm/RIU) }\end{array}$ & $\begin{array}{c}\text { Max. } \\
\text { Amplitude } \\
\text { sensitivity } \\
\text { (RIU-1) }\end{array}$ & $\begin{array}{c}\text { Max. } \\
\text { Wavelength } \\
\text { resolution } \\
\text { (RIU) }\end{array}$ & $\begin{array}{c}\text { Max. } \\
\text { Amplitude } \\
\text { resolution } \\
\text { (RIU) }\end{array}$ \\
\hline$[25]$ & $1.36-1.41$ & D-shaped & $\mathrm{Au} / \mathrm{TiO}_{2}$ & 30000 & - & $3.3 \times 10^{-6}$ & - \\
2019 & & & & & & & \\
\hline$[27]$ & $1.34-1.34$ & $\begin{array}{c}\text { Microchannel } \\
\text { based PCF }\end{array}$ & $\mathrm{AZO}$ & 5000 & 167 & $2 \times 10^{-5}$ & - \\
\hline 2018 & & & & & & \\
\hline$[28]$ & $1.36-1.40$ & $\begin{array}{c}\text { Circular } \\
\text { PCF }\end{array}$ & Niobium/ $\mathrm{Al}_{2} \mathrm{O}_{3}$ & 8000 & 1560 & $6.4 \times 10^{-6}$ & - \\
\hline
\end{tabular}




\begin{tabular}{|c|c|c|c|c|c|c|c|}
\hline $\begin{array}{l}{[29]} \\
2018\end{array}$ & $1.18-1.36$ & D-shaped & $\mathrm{Au}$ & 20000 & 1054 & $5 \times 10^{-6}$ & $6.7 \times 10^{-6}$ \\
\hline $\begin{array}{l}30] \\
2018\end{array}$ & $1.33-1.38$ & Spiral PCF & $\mathrm{Au}$ & 4600 & 420.4 & - & \\
\hline $\begin{array}{l}{[31]} \\
2019\end{array}$ & $\begin{array}{l}1.32-1.35 \\
1.36-1.40\end{array}$ & D-shaped & $\mathrm{Au} / \mathrm{Graphene}$ & $\begin{array}{c}5666 \\
33500\end{array}$ & - & $2.98 \times 10^{-5}$ & - \\
\hline $\begin{array}{l}{[34]} \\
2020\end{array}$ & $1.33-1.39$ & D-shaped & $\mathrm{Ag} / \alpha-\mathrm{Fe}_{2} \mathrm{O}_{3}$ & 6400 & - & - & - \\
\hline $\begin{array}{l}35] \\
2019\end{array}$ & $\begin{array}{l}1.33-1.36 \\
1.37-1.40\end{array}$ & D-shaped & $\begin{array}{l}\mathrm{Au} / \mathrm{MoS}_{2} / \\
\text { Graphene }\end{array}$ & $\begin{array}{c}6000 \\
14933.34\end{array}$ & - & $6.69 \times 10^{-6}$ & - \\
\hline $\begin{array}{l}{[36]} \\
2018\end{array}$ & $1.20-1.29$ & $\begin{array}{l}\text { Open-ring } \\
\text { based PCF }\end{array}$ & $\mathrm{Au}$ & 11055 & - & $9.05 \times 10^{-6}$ & - \\
\hline $\begin{array}{l}\text { This } \\
\text { Work }\end{array}$ & 1.27-1.39 & $\begin{array}{l}\text { SSP-PCF with } \\
\text { micro-opening }\end{array}$ & Au/antimonene & 77000 & 1320.41 & $\begin{array}{l}1.29 \\
\times 10^{-6}\end{array}$ & $\begin{array}{l}8.6 \\
\times 10^{-7}\end{array}$ \\
\hline
\end{tabular}

\section{Conclusions}

An ingenious, novel and practically approachable SPR based SSP-PCF sensor is presented by employing gold, and antimonene layers on the flat dual channel of the fiber. The analyte/biomolecules can be directly dropped onto the antimonene layer for sensing. The effect of air hole radius of the cladding, thickness of gold layer, and polishing height on the performance of sensor is also studied. The wavelength and amplitude sensitivities of the proposed sensor have reached $77000 \mathrm{nmRIU}^{-1}$ and $1320.41 \mathrm{RIU}^{-1}$, respectively. Aside from that, exceptionally fine wavelength and amplitude RI resolutions of $1.298 \times 10^{-6}$ RIU and $88.6 \times 10^{-7}$ RIU are attained, with a high FOM of $311.74 \mathrm{RIU}^{-1}$. The proposed sensor has covered low (below 1.33) as well as high RI detection range. Furthermore, proposed sensor has high sensitivity and FOM, therefore, it can be used for medicinal drugs, aerogel, halogenated organic acids, organic chemicals, and biological sensing.

Competing interest: The authors declare no conflict of interest.

Declarations: The authors have no relevant financial or non-financial interests to disclose. The authors have no conflicts of interest to declare.

\section{Acknowledgements}

Yogendra Kumar Prajapati gratefully acknowledges the DST-FIST, Govt. of India for the project (SR/FST/ETI418/2016).

\section{References}

Otupiri,R., Akowuah,E., Haxha,S.: Multi-channel SPR biosensor based on PCF for multi-analyte sensing applications Opt. Express 23 15716-15727 (2015)

Zhao, Y. Deng, Z.-Q., Li J.: Photonic crystal fiber based surface plasmon resonance chemical sensors Sens. Actuators B, Chem. 202 557-567(2014).

Hu D. J. J., Ho H. P.: Recent advances in plasmonic photonic crystal fibers: Design, fabrication and applications Adv. Opt. Photon $9257-314$ (2017) 
Chu, S. Nakkeeran, K., Abobaker, A.M., Aphale, S.S., Babu, P.R. Senthilnathan, K.: Design and analysis of surface-plasmonresonance-based photonic quasi-crystal fiber biosensor for high-refractive-index liquid analytes IEEE J. Sel. Top. Quantum Electron. 25 1-9 (2019).

Jabin, M.A., Ahmed, K., Rana, M.J., Paul, B.K., Luo, Y., Vigneswaran, D. :Titanium coated dual-core D-shaped SPR-based PCF for hemoglobin sensing Plasmonics 14 1601-1610(2019)

Huang T.: Highly sensitive SPR sensor based on D-shaped photonic crystal fiber coated with indium tin oxide at near-infrared wavelength Plasmonics 12 583-588(2017)

Singh, S., Prajapati, Y.K.:TiO $/$ Gold-Graphene Hybrid Solid Core SPR Based PCF RI Sensor for Sensitivity Enhancement Optik 224, 165525 (2020).

Li, Chenguang, Bei, Yan, Liu Jianjun : Refractive index sensing characteristics in a D-shaped photonic quasi-crystal fiber sensor based on surface plasmon resonance JOSA A 361663 (2019).

Haque, E., Hossain, M. Anwar, Namihira, Y., Ahmed F.: Microchannel-based plasmonic refractive index sensor for low refractive index detection Appl. Opt. 58 1547-1554 (2019).

Wang, F., Liu, C., Sun, Z., Sun, T., Liu, B., Chu: A highly sensitive spr sensors based on two parallel PCFs for low refractive index detection IEEE Photon. J. 10 7104010(2018)

Dash,J. N.,Jha,R.: Highly sensitive side-polished birefringent PCF based SPR sensor in near IR Plasmonics 11 1505-1509 (2016)

Wang, Shun, Li, Shuguang: Surface plasmon resonance sensor based on symmetrical side-polished dual-core photonic crystal fiber," Optical Fiber Technology 51 96-100 (2019).

Singh, S., Prajapati, Y.K.: Highly sensitive dual core symmetrical side polished modified D-shaped SPR based PCF refractive index sensor with deeply etched micro openings Optik 235, 166657 (2021).

Akter, Sanjida, Abdur Razza, S.M.:Highly sensitive open-channels based plasmonic biosensor in visible to near-infrared wavelength Results in Physics 13 (2019).

Chauhan, R. et al:: Label-free piezoelectric immunosensor decorated with gold nanoparticles: kinetic analysis and biosensing application Sens. Actuat. B Chem 222 804-816 (2016).

Novoselov, K. S., Geim, A. K., Morozov, S. V., Dubonos, S. V. et al. :Electric Field Effect in Atomically Thin Carbon Films Science, 306 666-669(2004).

Zhu, C, Du, D, Lin Y: Graphene and graphene-like 2D materials for optical biosensing and bioimaging: A review 2D Materials 2 032004 (2015).

$\mathrm{Gu}$, Xiaokun, Li, Baowe, Yang, Ronggui :Layer thickness- dependent phonon properties and thermal conductivity of $\mathrm{MoS}_{2}$ Journal of Applied Physics 119085106 (2016).

Wang, F, Kinloch, I A, Wolverson, D, Tenne, R, Zak, A, O'Connell, E, Bangert, U, Young, R J: Strain induced phonon shifts in tungsten disulfide nanoplatelets and nanotubes 2D Materials 4015007 (2017).

Lee, S., Esfarjani, K., Mendoza, J., Dresselhaus, M. S., Chen, G.: Lattice thermal conductivity of Bi, Sb, and Bi-Sb alloy from first principles Rev. B 89 085206(2014).

Wang, G., Pandey R., Karna, S. P.: Atomically Thin Group V Elemental Films: Theoretical Investigations of Antimonene Allotropes ACS Appl. Mater. Interfaces 711490 (2015).

Shao, Yan et al.: Epitaxial growth of flat antimonene monolayer: a new honeycomb analogue of graphene Nano Letters 18, 21332139 (2018).

Xue, T., Liang, W., Li, Y. et al.: Ultrasensitive detection of miRNA with an antimonene-based surface plasmon resonance sensor Nat Commun 10 (2019). https://doi.org/10.1038/s41467-018-07947-8.

Akowuah, E. K., Gorman, T., Ademgil, H., Haxha, S., Robinson, G. K., Oliver, J. V.: Numerical analysis of a photonic crystal fiber for biosensing applications," IEEE J. Quantum Electron. 48 1403-1410 (2012).

Gangwar, RK, Amorim, Vítor A., Marques, P.V.S.: High Performance Titanium oxide coated D-shaped Optical Fiber Plasmonic Sensor IEEE Sens. J.19 9244-9248 (2019).

Singh, Deobrat, Gupta, Sanjeev K., Sonvane, Yogesh et al: Antimonene: A monolayer material for ultra violet optical nanodevices J. Mater. Chem. C 4 6386-6390(2016).

Dash, J. N., Das, R., Jha, R. :AZO coated microchannel incorporated PCF-based SPR sensor: a numerical analysis IEEE photon Technol.Lett. 30 1032-1035 (2018).

Hasan, M. R., Akter, S., Ahmed, K., Abbott, D.: Plasmonic refractive index sensor employing niobium nanofilm on photonic crystal fiber IEEE Photon. Technol. Lett.30 315-318( 2018).

Haque, E., Hossain, M. A. Ahmed, F. Namihira, Y.: Surface plasmon resonance sensor based on modified D-shaped photonic crystal fiber for wider range of refractive index detection IEEE Sens. J.18 8287-8293 (2018). 
Hasan, M. R. et al. :Spiral photonic crystal fiber-based dual-polarized surface plasmon resonance biosensor," IEEE Sens. J.18, 133-140 (2018).

Singh,Shivam, Prajapati, Y.K.: Highly sensitive refractive index sensor based on D-shaped PCF with gold-graphene layers on the polished surface Applied Physics A 125 1-7 (2019).

Zakaria, R., Kam, W., Ong, Y. S. , Yusoff, S. F. A. Z., Ahmad H., Mohammed, Waleed S. :Fabrication and simulation studies on D-shaped optical fiber sensor via surface plasmon resonance Journal of Modern Optics 64 1443-1449 (2017).

Assebban, M., Gibaja, C., Fickert M. et al. :Unveiling the oxidation behavior of liquid-phase exfoliated antimony nanosheets 2D mater 7 1-24 (2020).

Kadhim, Riadh A., Yuan, Liming, Xu, Hao, Wu, Jiang, Wang, Zhiming :Highly sensitive D-shaped optical fibre surface plasmon resonance refractive index sensor based on $\mathrm{Ag}-\alpha-\mathrm{Fe}_{2} \mathrm{O}_{3}$ grating IEEE Sens. J. (2020), doi: DOI 10.1109/JSEN.2020.2992854.

Singh, S., Prajapati, Y.K.: Dual-polarized ultrahigh sensitive Gold/MoS $2 /$ Graphene based D-shaped PCF refractive index sensor in visible to near- IR region," Optical and Quantum Electronics 52 1-15(2019).

Chen Xin , Xia Li , Li, Chen :Surface Plasmon Resonance Sensor Based on a Novel D-Shaped Photonic Crystal Fiber for Low Refractive Index Detection IEEE Photonics Journal 10 ( 2018). 
